\title{
WIMP-nucleus scattering in chiral effective theory
}

\author{
Vincenzo Cirigliano $^{a}$, Michael L. Graesser ${ }^{a}$, and Grigory Ovanesyan ${ }^{a}$ \\ a Theoretical Division, Los Alamos National Laboratory \\ MS B283, Los Alamos, NM 87545, U.S.A.
}

\begin{abstract}
We discuss long-distance QCD corrections to the WIMP-nucleon(s) interactions in the framework of chiral effective theory. For scalar-mediated WIMP-quark interactions, we calculate all the next-to-leading-order corrections to the WIMP-nucleus elastic cross-section, including two-nucleon amplitudes and recoil-energy dependent shifts to the single-nucleon scalar form factors. As a consequence, the scalar-mediated WIMPnucleus cross-section cannot be parameterized in terms of just two quantities, namely the neutron and proton scalar form factors at zero momentum transfer, but additional parameters appear, depending on the short-distance WIMP-quark interaction. Moreover, multiplicative factorization of the cross-section into particle, nuclear and astro-particle parts is violated. In practice, while the new effects are of the natural size expected by chiral power counting, they become very important in those regions of parameter space where the leading order WIMP-nucleus amplitude is suppressed, including the so-called "isospin-violating dark matter" regime. In these regions of parameter space we find order-of-magnitude corrections to the total scattering rates and qualitative changes to the shape of recoil spectra.
\end{abstract}




\section{Introduction}

The evidence for Dark Matter (DM) is overwhelming. However currently all experimental evidence for its existence comes from astrophysical observations. Great progress has been made in last few years in laboratory underground experiments trying to measure elastic recoil of WIMPs (weakly interacting massive particles) from a nucleus inside the detector. The positive results reported by DAMA [1] and CoGeNT [2] are in contradiction with null results from XENON [3, 4] and CDMS [5, 6], when analyzed in a standard WIMP scenario [7, 8], and many ideas have been proposed to resolve this puzzle. These ideas mostly involve modifications to the short-distance dynamics, such as the inelastic dark matter scenario [9, 10, 11], isospin violating dark matter couplings (IVDM) [12, 13, 14, 15], momentum-dependent couplings [16, 17], and resonant scattering [18]. The impact of astrophysical uncertainties has also been explored in the recent literature [19, 20, 21, 22, 23].

Here we take a complementary point of view. We wish to study how known hadronic and nuclear physics effects (such as nucleon form factors and meson exchange currents) affect WIMP-nucleus elastic scattering, and explore whether the inclusion of these effects helps mitigate the apparent contradictions between DAMA, CoGeNT and XENON and CDMS. The need to revisit the hadronic and nuclear physics of WIMP-nucleus scattering has been pointed out in the recent literature, and first systematic studies in this direction have appeared in Refs. [24, 25]. Ref. [24] developed a non-relativistic effective field theory (EFT) in which the degrees of freedom are the WIMP and nucleus as a whole, and studied the set of distinguishable recoil spectra that could arise from different underlying models. The authors of Ref. [25], on the other hand, focused on a non-relativistic EFT at the WIMP-nucleon level, wrote the most general set of single-nucleon operators up to second order in momentum transfer, and worked out the corresponding nuclear responses (for relevant targets) within the nuclear shell model.

Our current work adds yet a different spin, in that it discusses the WIMP-nucleon interaction within the chiral EFT framework, which incorporates at the nucleon level the consequences of the broken chiral symmetry of QCD. For simplicity we discuss here only the case of scalarmediated WIMP-quark interaction and derive the leading order (LO) and next-to-leading order (NLO) WIMP-nucleon operators. To NLO, the resulting WIMP-nucleon operators involve not only the single nucleon (scalar) form factor, but also a two-nucleon operator, generated by a so-called meson-exchange diagram. The latter effect has been previously considered in Ref. [26], where it was claimed it could affect WIMP-nucleus cross-sections at the $\sim 50 \%$ level. Compared to the analysis of Ref. [26], we embed the meson-exchange diagram in a consistent chiral $S U(3)$ power counting to NLO, that includes $\eta-\eta$ exchange in addition to $\pi-\pi$ exchange and also loop corrections to the one-nucleon amplitudes (not considered in [26]). Compared to Ref. [25] our work considers a more restricted underlying interaction by assuming that WIMP interacts with quarks via the scalar density. Within this restricted setting, however, we go beyond Ref. [25] in several respects: (i) Working to NLO in the ratio of momentum transfer to nucleon mass, we include all the interactions consistent with QCD. These include form-factor corrections to the one-nucleon operators (which are left unspecified in Ref. [25]) as well as two-nucleon operators not considered in Ref. 25]. To our knowledge this is the first time the chiral power corrections are consistently included for WIMP-nucleus elastic cross-section. (ii) Because we start from the underlying WIMP-quark interaction, we are able to relate the co- 
efficients of the WIMP-nucleon operators (left arbitrary in the bottom-up nucleon-level EFT approach of [25]) to the short-distance parameters of the theory.

The paper is organized as follows. In Section 2 we set up the framework for our discussion, specifying the short-distance scalar-mediated WIMP-quark interaction in a model-independent way. In Section 3 we discuss the chiral perturbation theory (ChPT) framework and identify the LO and NLO graphs that contribute to scalar-mediated WIMP scattering off nucleons. In Section 4 we compute the relevant NLO corrections, that include one loop diagrams (4.1) twobody meson-exchange diagrams 4.2 . In Section 5 we discuss the matrix elements of WIMPnucleon operators in the nucleus, and briefly review the nuclear shell model (NSM) that we use to evaluate the matrix elements of two-body operators. We consider the phenomenological implications of our NLO corrections in Section 6, and conclude in section 7 .

\section{Scalar-mediated WIMP-quark interaction}

We consider the following model-independent interaction between WIMP and quarks:

$$
\mathcal{L}_{\chi q}=\sum_{q=u, d, s, c, b, t} k_{q} m_{q} \bar{q} q+k_{G} \frac{\alpha_{s}}{\pi} G_{\mu \nu} G^{\mu \nu},
$$

where $k_{q, G}$ are functions of the DM field(s). For example, in the case of Dirac or Majorana fermion DM one has $k_{q, G} \propto \bar{\chi} \chi$.Integrating out the heavy quarks $c, b, t$ [27] we get the following effective interaction:

$$
\mathcal{L}_{\text {eff }}=\sum_{q=u, d, s} s_{q} \bar{q} q+s_{\Theta} \Theta_{\mu}^{\mu}
$$

where we have used the trace anomaly equation and $\Theta_{\mu}^{\mu}$ is the trace of the stress energy tensor. The fields $s_{q}$ and $s_{\Theta}$ are related to $k_{q, G}$ as follows:

$$
s_{q}=m_{q}\left(k_{q}-\frac{2}{27} \sum_{Q=c, b, t} k_{Q}+\frac{8}{9} k_{G}\right), \quad s_{\Theta}=\frac{2}{27} \sum_{Q=c, b, t} k_{Q}-\frac{8}{9} k_{G} .
$$

Specializing to the case of fermionic DM particle (denoted by $\chi$ ), we can write the fields $k_{q, G}$ in terms of $\chi$ and dimensionless short-distance parameters $\tilde{\lambda}_{q, G}$

$$
k_{q}=\frac{\tilde{\lambda}_{q}}{v \Lambda_{\mathrm{np}}^{2}} \bar{\chi} \chi, \quad k_{G}=\frac{\tilde{\lambda}_{G}}{v \Lambda_{\mathrm{np}}^{2}} \bar{\chi} \chi
$$

where $v=\left(\sqrt{2} G_{F}\right)^{-1 / 2}$ is the Higgs VEV and $\Lambda_{\text {np }}$ denotes a generic new-physics scale. After integrating out the heavy quarks, the low-energy effective Lagrangian (2) contains only four dimensionless parameters $\lambda_{u, d, s, \Theta}$ :

$$
s_{q}=\frac{m_{q}}{v} \frac{\lambda_{q}}{\Lambda_{\mathrm{np}}^{2}} \bar{\chi} \chi \quad s_{\Theta}=\frac{\lambda_{\Theta}}{v \Lambda_{\mathrm{np}}^{2}} \bar{\chi} \chi
$$



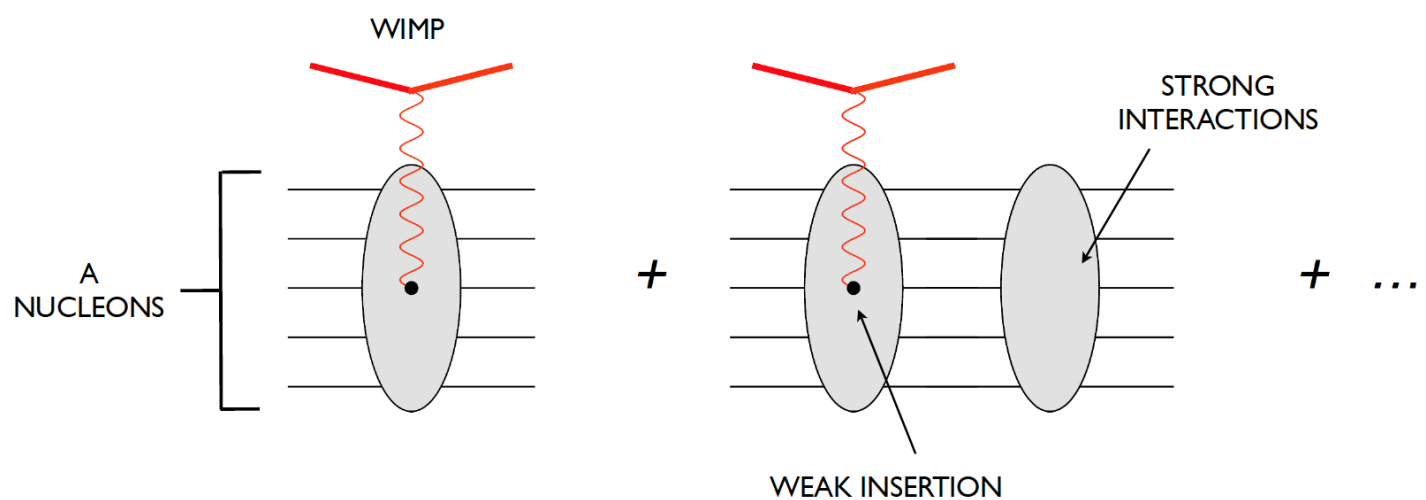

Figure 1: Representation of ladder diagrams contributing to $T_{A, W}$, the scattering amplitude for $A$ nucleons and a WIMP. The ladder rungs are given by $A$-nucleon irreducible amplitudes; only one of the rungs (denoted by $M_{A, W}$ in the text) involves a WIMP scalar density insertion.

In our phenomenological analysis we will use $\lambda_{u, d, s, \Theta}$, but the reader should keep in mind that these are related to the short distance parameters by $\lambda_{q}=\tilde{\lambda}_{q}-(2 / 27) \sum_{Q} \tilde{\lambda}_{Q}+(8 / 9) \tilde{\lambda}_{G}$ and $\lambda_{\Theta}=(2 / 27) \sum_{Q} \tilde{\lambda}_{Q}-(8 / 9) \tilde{\lambda}_{G}$.

In order to compute the WIMP-nucleus cross-sections from the short-distance Lagrangian of Eq. (1) that couples WIMPs to quarks, two steps are needed: (i) At an energy scale of the order of $\sim \mathrm{GeV}$ one matches the WIMP-quark Lagrangian non-perturbatively to a WIMPnucleon effective Lagrangian. For this purpose we use ChPT [28, 29, 30] (for reviews, see [31] and [32]), which parameterizes the non-perturbative physics in a number of low-energy constants that can either be determined phenomenologically or computed in lattice QCD [33]. This step provides an effective potential describing the interaction of the WIMP with nucleons. (ii) With the WIMP-nucleon interaction Hamiltonian at hand, one then calculates the amplitude for WIMP-nucleus elastic scattering. This step requires information on the wavefunction of the target nucleus in the ground state. The WIMP-nucleus interaction can also be parameterized in terms of a non-relativistic effective Lagrangian [24]. Note that while for definiteness we are focusing here only on the case of scalar-mediated WIMP-quark interactions, a similar analysis can be performed for all types of quark-WIMP interactions (vector, axialvector, pseudoscalar, tensor) and will be presented elsewhere [34].

\section{WIMP-nucleon interactions in chiral effective theory}

Given the kinematics of WIMP-nucleus elastic scattering, the three-momentum transfer to the hadronic system does not exceed $q_{\max }=2 \mu_{W A} v_{\text {rel }}<2 m_{A} v_{0} \sim 200 \mathrm{MeV}$, where $\mu_{W A}$ is the WIMP-nucleus reduced mass and we have used a typical target nucleus mass $m_{A} \sim 100 \mathrm{GeV}$ as well as $v_{0} \sim 10^{-3}$ for the value at which the velocity distribution of the dark matter halo starts to fall off exponentially. Since $q_{\max }$ is small compared to the nucleon mass $m_{N}$, it is 
appropriate to use ChPT to describe the WIMP-nucleon dynamics, expanding the amplitudes in $p \sim q / m_{N} \sim m_{\pi, K, \eta} / m_{N}$.

As suggested by the form of Eq. (2), the weak interaction of WIMPs and light quarks can be incorporated in the framework of ChPT through the external source method [29], i.e. adding to the QCD Lagrangian external scalar sources $s(x)=\operatorname{diag}\left(s_{u}(x), s_{d}(x), s_{s}(x)\right)$ coupled to the scalar quark density and the external source $s_{\Theta}(x)$ coupled to the energy-momentum tensor. Note that from the point of view of chiral symmetry, the external scalar source transforms in the same way as the quark mass matrix $m_{q}$. The octet of light pseudoscalar meson fields $\left(\phi_{a}\right)$ is described by the matrix $U=\operatorname{Exp}\left(i \sum_{a} T_{a} \phi_{a} / F\right)$, where $F$ can be identified to leading order with the pion decay constant and $T_{a}$ are the $S U(3)$ generators. In the chiral power counting one assigns the following scaling: $\partial U \sim O(p)$, while $m_{q} \sim s \sim O\left(p^{2}\right)$ and $s_{\Theta} \sim O\left(p^{0}\right)$. In terms of these fields, the lowest order mesonic chiral Lagrangian reads

$$
\begin{aligned}
& \mathcal{L}_{M}=\mathcal{L}_{M}^{(2)}+\mathcal{L}_{M}^{(4)}+\ldots \quad \mathcal{L}_{M}^{(2 n)} \sim O\left(p^{2 n}\right), \\
& \mathcal{L}_{M}^{(2)}=\frac{F^{2}}{4} \operatorname{Tr}\left[\partial_{\mu} U^{\dagger} \partial^{\mu} U\right]+\frac{B_{0} F^{2}}{2} \operatorname{Tr}\left[\left(m_{q}-s\right)\left(U+U^{\dagger}\right)\right] .
\end{aligned}
$$

The low-energy constant (LEC) $B_{0}$ is related to the light quark condensate: $\langle\bar{q} q\rangle=-F^{2} B_{0}(1+$ $O\left(m_{q}\right)$ ). For the meson-baryon Lagrangian we use the Heavy Baryon Chiral Perturbation Theory formulation [35], in which one writes the baryon momentum as $p_{B}=m v+k$, in terms of a velocity $v_{\mu}$ and a residual momentum $k \sim O(p)$ and one re-defines the heavy baryon octet field $B$ such that $\partial B \sim O(p)$. Introducing the covariant derivative $\nabla_{\mu} B=\partial_{\mu} B+\left[\Gamma_{\mu}, B\right]$ with $\Gamma_{\mu}=1 / 2\left(u^{\dagger} \partial_{\mu} u+u \partial_{\mu} u^{\dagger}\right)$, the spin vector $S_{\mu}=(i / 2) \gamma_{5} \sigma_{\mu \nu} v^{\nu}$, the field $u_{\mu}=i\left(u^{\dagger} \partial_{\mu} u-u \partial_{\mu} u^{\dagger}\right)$ with $u=\sqrt{U}$, and finally $\chi_{+}=u^{\dagger} \chi u^{\dagger}+u \chi^{\dagger} u$ with $\chi=2 B_{0}\left(m_{q}-s\right)$, the first few terms in the chiral expansion read [35, 31]

$$
\begin{aligned}
& \mathcal{L}_{M B}=\mathcal{L}_{M B}^{(1)}+\mathcal{L}_{M B}^{(2)}+\ldots \quad \mathcal{L}_{M}^{(n)} \sim O\left(p^{n}\right) \\
& \left.\mathcal{L}_{M B}^{(1)}=\operatorname{Tr}(\bar{B} i v \cdot \nabla B)+D \operatorname{Tr}\left(\bar{B} S^{\mu}\left\{u_{\mu}, B\right\}\right)+F \operatorname{Tr}\left(\bar{B} S^{\mu}\left[u_{\mu}, B\right]\right]\right) \\
& \mathcal{L}_{M B}^{(2)}=b_{D} \operatorname{Tr}\left(\bar{B}\left\{\chi_{+}, B\right\}\right)+b_{F} \operatorname{Tr}\left(\bar{B}\left[\chi_{+}, B\right]\right)+b_{0} \operatorname{Tr}(\bar{B} B) \operatorname{Tr}\left(\chi_{+}\right)
\end{aligned}
$$

In the above expression $F, D, b_{F}, b_{D}, b_{0}$ are low-energy constants, related to the baryon axial current matrix elements $(F, D)$, and the baryon mass splitting and sigma-terms $\left(b_{F}, b_{D}, b_{0}\right)$.

The EFT power counting allows one to identify the leading contributions to the scattering amplitude $T_{A, W}$ for the process $N_{1}+\cdots+N_{A}+W \longrightarrow N_{1}+\cdots+N_{A}+W$ involving $A$ nucleons and a WIMP. As is well known [36, 37, 38], when $A>1$ the naive chiral power counting breaks down due to pinch singularities that arise when nucleons in the intermediate state simultaneously go on shell. As illustrated in Fig. 1, the full non-perturbative amplitude $T_{A, W}$ is obtained by summing a Lippmann-Schwinger series of ladder diagrams with $A$-nucleons intermediate states and rungs given by $A$-nucleon irreducible amplitudes (not necessarily connected), only one of which involves the insertion of the external probe (the WIMP scalar density in the case at hand). These $A$-nucleon irreducible amplitudes admit a consistent power counting, and the scaling of the full amplitude $T_{A, W}$ is controlled by the scaling of $M_{A, W}$, the $A$-nucleon irreducible amplitude with insertion of the external probe. 


\begin{tabular}{|c|c|c|c|}
\hline Effective Lagrangian & $d_{i}$ & $n_{i}$ & $\epsilon_{i} \equiv d_{i}+\frac{n_{i}}{2}-2$ \\
\hline $\mathcal{L}_{M}^{(2 n)}$ & $2 n$ & 0 & $2(n-1)$ \\
\hline $\mathcal{L}_{M B}^{(n)}$ & $n$ & 2 & $n-1$ \\
\hline
\end{tabular}

Table 1: Chiral dimensions for vertices arising from the purely mesonic and baryon-meson effective Lagrangians. $n=1,2, \ldots$ represents any positive integer.

A diagram with $C$ connected parts, $L$ loops, $V_{i}$ strong-interactions vertices of type $i$ and one "weak" vertex scales as $M_{A, W} \sim p^{\nu}$ with [37, 32]

$$
\nu=4-A-2 C+2 L+\sum_{i} V_{i} \epsilon_{i}+\epsilon_{W} .
$$

The effective chiral dimension of vertex $i$ is given by $\epsilon_{i}=d_{i}+n_{i} / 2-2 \geq 0$, where $d_{i}$ is the chiral dimension of the vertex (e.g. a vertex from $\mathcal{L}_{M B}^{(1)}$ has $d_{i}=1$ ), and $n_{i}$ is the number of baryonic legs attached to the vertex. Note that in Eq. (11) we have explicitly isolated the contribution $\epsilon_{W}$ due to the weak vertex involving the external source coupled to the WIMP. In Table 1 we give a summary of chiral dimensions for the relevant effective Lagrangians.

For fixed $A$, the leading contributions to the amplitude are obtained by minimizing $\nu$ in Eq. (11), which is obtained by: (i) maximizing the number of connected contributions $C=A, A-1, \ldots$; (ii) minimizing the number of loops $L=0,1, \ldots$; (iii) using strong vertices from the lowest order Lagrangians $\mathcal{L}_{M}^{(2)}$ and $\mathcal{L}_{M B}^{(1)}\left(\epsilon_{i}=0\right.$ ), so as to minimize the $\epsilon_{i}$; (iv) attaching the external scalar source to a baryon line using a vertex from $\mathcal{L}_{M B}^{(2)}\left(\epsilon_{W}=1\right)$ or to a meson line using a vertex from $\mathcal{L}_{M}^{(2)}\left(\epsilon_{W}=0\right)$, consistently with the requirement that there are no external meson lines and the choice of $C$ and $L$. In the case of external scalar source we find:

- The leading order diagrams have $C=A, L=0$, and $\epsilon_{W}=1$ 1.e. they have $A$ disconnected parts, no loops, (i.e. no mesons in the diagram), and the source attached to one of the nucleon lines through the vertex in $\mathcal{L}_{M B}^{(2)}$. This corresponds to $\nu_{\mathrm{LO}}=5-3 A$.

- Three classes of diagrams can contribute to NLO $\left(\nu=\nu_{\mathrm{LO}}+1\right)$ as can be seen by inspecting Eq. (11): (i) $C=A, L=1, \epsilon_{W}=0$, i.e. diagrams with $A$ disconnected parts, one of which involves a one-loop diagram with vertices from $\mathcal{L}_{M B}^{(1)}$ and the source attached to a meson line through $\mathcal{L}_{M}^{(2)}$ (see Fig. 2); (ii) $C=A-1, L=0, \epsilon_{W}=0$, i.e. diagrams with $A-1$ disconnected parts, one of which involves two nucleon lines connected by meson exchange with vertices from $\mathcal{L}_{M B}^{(1)}$ and source attached to the meson line through $\mathcal{L}_{M}^{(2)}$ (see Fig. 3); (iii) $C=A, L=0, \epsilon_{W}=2$, i.e. same topology as the leading diagram but with the source attached to a nucleon line through a vertex from the $O\left(p^{3}\right)$ Lagrangian $\mathcal{L}_{M B}^{(3)}$. By inspecting of the only relevant vertex [31] $\mathcal{L}_{M B}^{(3)} \supset \operatorname{Tr}\left(\chi_{+}\right) \operatorname{Tr}(\bar{B} v \cdot \partial B)$, one

\footnotetext{
${ }^{1}$ Note that $\epsilon_{W}=0$ is not consistent with the choice $C=A$ and $L=0$, because the source would have to couple to a meson line and the meson line has to attach to nucleons. This produces either $L=1$ or $C=A-1$.
} 

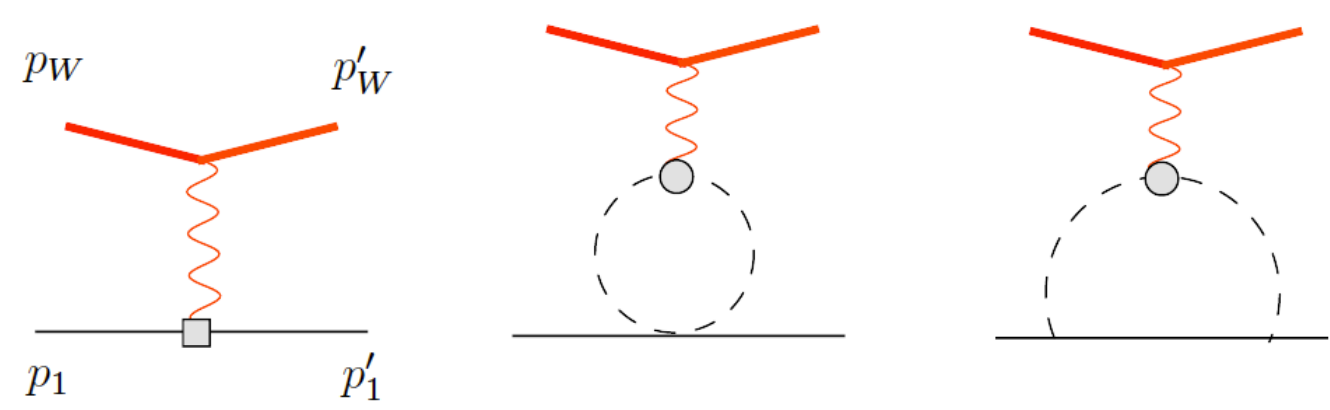

Figure 2: LO and NLO diagrams contributing $M_{1, W}$. Black solid (dashed) lines denote nucleons (mesons).

sees that for on-shell nucleons this contribution actually scales as of $p^{\nu_{\mathrm{LO}}+2}$ and therefore enters at NNLO.

The number of diagrams grows quickly as one goes beyond NLO, and higher order terms will involve in general vertices from effective Lagrangians containing more than two baryon fields, not considered in Table 1. In this work we consider only NLO contributions and note that a consistent chiral counting to NLO requires to include not only loop corrections to the nucleon scalar form factors (Fig. 2), but also meson-exchange diagrams that result in two-nucleon operators (Fig. 3)

Finally, a similar analysis can be done for the insertion of the energy-momentum tensor vertices, coupled to the external source $s_{\Theta}(x)$ (see Eq. (2p). Using the observation that insertions of the energy-momentum tensor on a baryon line scale as $\Theta_{\mu}^{\mu} \sim O\left(p^{0}, p, \ldots\right)$ [34] (corresponding to $\left.\epsilon_{W}=-1,0, \ldots\right)$ and on a meson line as $\Theta_{\mu}^{\mu} \sim O\left(p^{2}, p^{4}, \ldots\right)$ [39] (corresponding to $\left.\epsilon_{W}=0,2, \ldots\right)$, we find that the first chiral corrections to the relation $\left\langle N\left|\Theta_{\mu}^{\mu}\right| N\right\rangle=m_{N} \bar{\psi}_{N} \psi_{N}$ arise in principle at NNLO. Moreover, an explicit calculation [34. shows that the relevant diagrams cancel to this order, thus pushing the corrections to $\mathrm{N}^{3} \mathrm{LO}$.

\section{$4 \quad$ NLO corrections}

We now discuss the NLO contributions to $M_{A, W}$, the $A$-nucleon irreducible amplitude in presence of one insertion of the external source. As discussed earlier, the NLO corrections fall into two classes: loop diagrams contributing to single-nucleon amplitudes and tree-level diagrams contributing to two-nucleon interactions.

\subsection{One-nucleon amplitude}

The one-nucleon amplitude starts at leading order (tree-level diagram with vertex from $\mathcal{L}_{M B}^{(2)}$ in Eq. (10) above) and receives NLO corrections through one-loop diagrams, as shown in Figure 2. Including NLO corrections, and denoting the HBChPT spinors for proton and neutron with $N^{T}=\left(H_{v}^{(p)}, H_{v}^{(n)}\right)$, the one-nucleon amplitude reads

$$
M_{1, W}=\bar{\chi} \chi\left[\frac{1}{2}\left(f_{p}\left(q^{2}\right)+f_{n}\left(q^{2}\right)\right) \bar{N} N+\frac{1}{2}\left(f_{p}\left(q^{2}\right)-f_{n}\left(q^{2}\right)\right) \bar{N} \tau_{3} N\right]
$$


with proton and neutron form factors given by

$$
f_{p / n}\left(q^{2}\right)=\frac{1}{v \Lambda_{\mathrm{np}}^{2}}\left[\sum_{q=u, d, s} \lambda_{q} \sigma_{q}^{(p / n)}+\lambda_{\Theta} m_{p / n}-\frac{g_{A}^{2}}{64 \pi F_{\pi}^{2}}\left(A\left(q^{2}\right) \pm B\left(q^{2}\right)\right)\right],
$$

where $A(0)=B(0)=0 . f_{p / n}(0)$ receive contributions to LO (in terms of the couplings $b_{0}, b_{D}, b_{F}$ appearing in Eq. (10) ) and NLO (loops) in the chiral expansion. Here we have chosen to lump these contributions in the sigma-terms defined by $\left\langle i\left|m_{q} q \bar{q}\right| i\right\rangle=\sigma_{q}^{(i)} \bar{\psi}_{i} \psi_{i}$.

The $\sigma_{q}^{(i)}(i=p, n)$ can be expressed in terms of the matrix elements $\sigma_{\pi N}=\left(\left(m_{u}+\right.\right.$ $\left.\left.m_{d}\right) / 2\right)\langle p|\bar{u} u+\bar{d} d| p\rangle, \xi=\langle p|\bar{u} u-\bar{d} d| p\rangle /\langle p|\bar{u} u+\bar{d} d| p\rangle, y=2\langle p|\bar{s} s| p\rangle /\langle p|\bar{u} u+\bar{d} d| p\rangle$, and ratios of the light quark masses (see for example [40]). $\sigma_{\pi N}$ and $y$ can be extracted phenomenologically from baryon masses and meson-baryon scattering data [41, 42] or can be computed within lattice QCD [43, 44, 45, 46, 47, 48] (see Ref. [33] for a recent review), while $\xi$ can be related to $y$ through an analysis of baryon masses in the $S U(3)$ limit [49]. In our analysis we use the same relations of Ref. [40], but with updated numerical input on $\sigma_{\pi N}, \sigma_{s}^{(p)}$ (for which we use the ranges $\sigma_{\pi N}=(45 \pm 15) \mathrm{MeV}$ and $\sigma_{s}^{(p)}=(45 \pm 25) \mathrm{MeV}$ [33] $)$ and the ratios of quark masses (for which we use the PDG values [50]).

The momentum-dependent part of the form factors arise to this order entirely from the one-loop diagrams, and depend on the lowest order couplings $F, D$ of $\mathcal{L}_{M B}^{(1)}$ in Eq. (9) through the combinations $g_{A}=D+F=1.27$ [50] and $\alpha=F /(D+F) \approx 0.4$ [41] (for which we will use the range $\alpha \in[0.3,0.5])$. Using the on-shell condition for external heavy baryons, the diagrams in Figure 2 are finite. Defining $\lambda_{ \pm} \equiv\left(m_{u} \lambda_{u} \pm m_{d} \lambda_{d}\right) /\left(m_{u}+m_{d}\right)$ and $x_{M}=-q^{2} / m_{M}^{2}$, the form factors are given by: ${ }^{2}$

$$
\begin{aligned}
A\left(q^{2}\right) & =3 m_{\pi}^{3} \lambda_{+} \bar{f}\left(x_{\pi}\right)+\left(\frac{m_{\pi}^{2}}{3} \lambda_{+}+\frac{4\left(m_{K}^{2}-\frac{1}{2} m_{\pi}^{2}\right)}{3} \lambda_{s}\right)\left(\frac{1-4 \alpha}{\sqrt{3}}\right)^{2} m_{\eta} \bar{f}\left(x_{\eta}\right) \\
& +\left(\frac{m_{\pi}^{2}}{2} \lambda_{+}+\left(m_{K}^{2}-\frac{1}{2} m_{\pi}^{2}\right) \lambda_{s}\right)\left(3(1-2 \alpha)^{2}+\left(\frac{1+2 \alpha}{\sqrt{3}}\right)^{2}\right) m_{K} \bar{f}\left(x_{K}\right) \\
B\left(q^{2}\right) & =m_{\pi}^{2} \lambda_{-}\left[\frac{1}{2}\left(\left(\frac{2 \alpha+1}{\sqrt{3}}\right)^{2}-(1-2 \alpha)^{2}\right) m_{K} \bar{f}\left(x_{K}\right)\right. \\
& \left.-\frac{1-4 \alpha}{3}\left(m_{\eta}+m_{\pi}\right) \bar{f}_{1}\left(x_{\eta}, x_{\pi}\right)\right] .
\end{aligned}
$$

The loop functions describing the running of mesons with equal or different masses inside the loops are given by $\bar{f}(x)=f(x)-f(0)$ and $\bar{f}_{1}\left(x_{1}, x_{2}\right)=f_{1}\left(x_{1}, x_{2}\right)-4\left(1+z+z^{2}\right) /(1+z)^{2}$ $\left(z=m_{\pi} / m_{\eta}\right)$, respectively, with

$$
f(x)=2+\frac{(2+x)}{\sqrt{x}} \operatorname{ArcCot}\left(\frac{2}{\sqrt{x}}\right),
$$

\footnotetext{
${ }^{2}$ We use the leading-order mass relations with $m_{u}=m_{d}$ to express the products $B_{0} m_{q}$ in terms of meson masses $m_{\pi, K, \eta}$. On the other hand, we keep $m_{u} \neq m_{d}$ in the overall factors $\lambda_{ \pm}$. This prescription allows us to keep terms of order $\left(m_{u}-m_{d}\right) /\left(m_{u}+m_{d}\right) \sim O(1)$, while neglecting terms of order $\left(m_{u}-m_{d}\right) / m_{s} \ll 1$.
} 


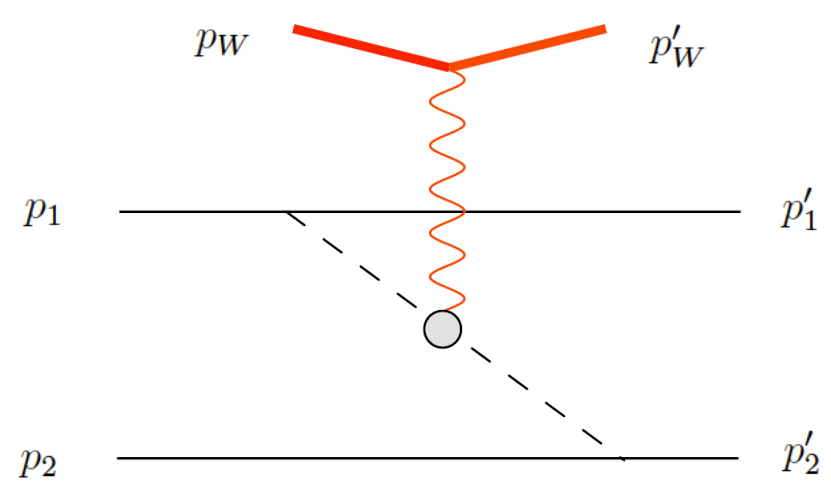

Figure 3: Tree-level diagram contributing to $M_{2, W}$. Black solid (dashed) lines denote nucleons (mesons).

$$
f_{1}\left(x_{1}, x_{2}\right)=2+\frac{\left(x_{1}+x_{2}+x_{1} x_{2}\right)\left(\operatorname{ArcCot}\left[\frac{2 \sqrt{x_{1}} x_{2}}{x_{1}+x_{1} x_{2}-x_{2}}\right]+\operatorname{ArcCot}\left[\frac{2 \sqrt{x_{2}} x_{1}}{x_{2}+x_{1} x_{2}-x_{1}}\right]\right)}{x_{1} \sqrt{x_{2}}+\sqrt{x_{1}} x_{2}},
$$

and have the following useful properties: $f(x \rightarrow 0)=3+\frac{5 x}{12}+\mathcal{O}\left(x^{2}\right), f_{1}(x, x) \equiv f(x)$.

\subsection{Two-nucleon amplitude}

As was derived in Section 3, at NLO there appears also a contribution with $A-1$ tree-level disconnected nucleon sectors, one of which involves two nucleons and the external source. The relevant diagram is shown in Figure 3 , and the possible mesons that are exchanged are limited to $\pi$ and $\eta$. The corresponding "direct" connected amplitude reads $\left(q_{i}=p_{i}-p_{i}^{\prime}\right.$ denotes the four-momentum transfer for each nucleon):

$$
\begin{aligned}
& M_{2, W}=\mathcal{M}_{\pi \pi}+\mathcal{M}_{\eta \eta} \\
& \mathcal{M}_{\pi \pi}=-\frac{1}{v \Lambda_{\mathrm{np}}^{2}} \frac{g_{A}^{2}}{F_{\pi}^{2}} \frac{m_{\pi}^{2} \lambda_{+}}{\left(q_{1}^{2}-m_{\pi}^{2}\right)\left(q_{2}^{2}-m_{\pi}^{2}\right)} \bar{N} q_{1} \cdot S \tau_{1}^{k} N \bar{N} q_{2} \cdot S \tau_{2}^{k} N \bar{\chi} \chi \\
& \mathcal{M}_{\eta \eta}=-\frac{1}{v \Lambda_{\mathrm{np}}^{2}} \frac{g_{A}^{2}}{3 F_{\pi}^{2}}\left(\frac{4 \alpha-1}{\sqrt{3}}\right)^{2} \frac{m_{\pi}^{2} \lambda_{+}+4\left(M_{K}^{2}-\frac{1}{2} m_{\pi}^{2}\right) \lambda_{s}}{\left(q_{1}^{2}-m_{\eta}^{2}\right)\left(q_{2}^{2}-m_{\eta}^{2}\right)} \bar{N} q_{1} \cdot S N \bar{N} q_{2} \cdot S N \bar{\chi} \chi
\end{aligned}
$$

There is also an "exchange" amplitude, which is obtained from the direct one by changing the overall sign and interchanging all variables of the final-state nucleons $\left(p_{1}^{\prime} \leftrightarrow p_{2}^{\prime}, N_{1}^{\prime} \leftrightarrow N_{2}^{\prime}\right)$.

Compared to Ref. [26], where the $\pi \pi$ two-body interaction has been calculated, we also include the $\eta \eta$ which is Yukawa enhanced as can be seen from Eq. 20). Later on we will study the competition between this enhancement and the suppression expected from the fact that the $\eta$-induced potential has shorter range compared to the $\pi$-mediated one. 


\subsection{WIMP-nucleon potentials}

From the WIMP-nucleon amplitudes discussed above to NLO in the chiral power counting, one can derive non-relativistic WIMP-nucleon interaction potentials. This procedure is standard and requires (i) taking the non-relativistic limit of $M_{1, W}$ and $M_{2, W}$ and rescaling the amplitude to take into account the difference between relativistic and non-relativistic normalization of states: $M_{A, W} \rightarrow \bar{M}_{A, W} \equiv 1 /\left.\left(\left(2 m_{N}\right)^{A}\left(2 m_{W}\right)\right) M_{A, W}\right|_{\text {non-rel }}$; (ii) determining the potential by requiring that the amplitude of non-relativistic quantum mechanics matches $\bar{M}_{A, W}$, using the same (unbound) WIMP and nucleon external states used in the ChPT calculation. This step involves taking the Fourier transform of $\bar{M}_{A, W}\left(\vec{q}_{i}, \vec{q}_{W}\right)$. Note that for the two body term one can work exclusively with the "direct" amplitude and match it on the QM matrix element of the potential between nucleon wave-functions that are not anti-symmetrized. The same result would be obtained by including the "exchange" diagram and using an anti-symmetric two-body wave-function.

The matching procedure leads to a QM interaction Hamiltonian of the form:

$$
H_{I}=\sum_{i=1, A} V_{1}\left(\vec{x}_{i}-\vec{x}_{W}\right)+\sum_{i<j} V_{2}\left(\vec{x}_{i}-\vec{x}_{W}, \vec{x}_{j}-\vec{x}_{W}\right)+\ldots,
$$

where the 1-body $\left(V_{1}\right)$ and 2-body $\left(V_{2}\right)$ terms are related to $\bar{M}_{1, A}$ and $\bar{M}_{2, A}$, respectively. The LO matching induces a contact one-body potential $V_{1}\left(\vec{x}_{i}-\vec{x}_{W}\right) \propto \delta^{(3)}\left(\vec{x}_{i}-\vec{x}_{W}\right)$. The NLO matching induces a long-range term in the WIMP-nucleon potential $V_{1}\left(\vec{x}_{i}-\vec{x}_{W}\right)$, as well as a contribution to the WIMP-nucleon-nucleon potential. As discussed below, we shall not need the detailed expression of the coordinate-space potentials, but rather a hybrid form that depends on the nucleon coordinates $\vec{x}_{i}$ (and spin-isospin variables) and the WIMP momentumtransfer variable $\vec{q}_{W}$.

\section{$5 \quad$ Nuclear matrix elements}

The next step towards a description of WIMP-nucleus elastic scattering requires using the WIMP-nucleon(s) interaction Hamiltonian (21) to compute the transition amplitude between appropriate initial and final WIMP-nucleus states. The initial state $|i\rangle$ is characterized by a WIMP with momentum $\vec{P}_{W}$ (and possibly internal quantum numbers) and nucleus in its ground state with center-of-mass momentum $\vec{P}_{A}$. The final state $|f\rangle$ is characterized by momenta $\vec{P}_{W}^{\prime}=\vec{P}_{W}-\vec{q}_{W}$ and $\vec{P}_{A}^{\prime}=\vec{P}_{A}-\vec{q}_{A}$, with the nucleus remaining in the ground state. Using translational invariance, the nucleus wave-function in the initial state can be written as $\psi_{i}\left(\vec{x}_{1}, \ldots, \vec{x}_{A}\right)=e^{i \vec{P}_{A} \cdot \vec{X}_{C M}} \phi_{0}\left(\vec{y}_{1}, \ldots, \vec{y}_{A-1}\right)$, where for simplicity we have suppressed spin and isospin indices, $\vec{X}_{C M}, \vec{y}_{1}, \ldots, \vec{y}_{A-1}$ represent center-of-mass and internal (Jacobi) coordinates for the system of $A$ nucleons, and $\phi_{0}\left(\vec{y}_{1}, \ldots, \vec{y}_{A-1}\right)$ is the ground-state wave-function in internal coordinates.

Within this setup, one can show [34] that the non-relativistic $T$-matrix element reads

$$
\langle f|\hat{T}| i\rangle=(2 \pi)^{3} \delta^{(3)}\left(\vec{q}_{W}+\vec{q}_{A}\right) T\left(\vec{q}_{W}\right) \quad T\left(\vec{q}_{W}\right)=T_{1}+T_{2}
$$




$$
\begin{aligned}
& T_{1}=\sum_{i=1, A} \int d \vec{x}_{1} \ldots d \vec{x}_{A} \psi_{0}^{*}\left(\vec{x}_{1}, \ldots, \vec{x}_{A}\right) \otimes \tilde{V}_{1}\left(\vec{q}_{W} ; \vec{x}_{i}\right) \otimes \psi_{0}\left(\vec{x}_{1}, \ldots, \vec{x}_{A}\right) \\
& T_{2}=\sum_{i<j} \int d \vec{x}_{1} \ldots d \vec{x}_{A} \psi_{0}^{*}\left(\vec{x}_{1}, \ldots, \vec{x}_{A}\right) \otimes \tilde{V}_{2}\left(\vec{q}_{W} ; \vec{x}_{i}, \vec{x}_{j}\right) \otimes \psi_{0}\left(\vec{x}_{1}, \ldots, \vec{x}_{A}\right),
\end{aligned}
$$

where we indicate with the symbol " $\otimes$ " the non-trivial contractions in spin and isospin space. In the above expressions $\psi_{0}\left(\vec{x}_{1}, \ldots, \vec{x}_{A}\right)$ denotes the ground-state nuclear wave-function with center-of-mass localized at the origin, i.e. $\psi_{0}\left(\vec{x}_{1}, \ldots, \vec{x}_{A}\right)=\psi_{C M}\left(\vec{X}_{C M}\right) \phi_{0}\left(\vec{y}_{1}, \ldots, \vec{y}_{A-1}\right)$, with $\left|\psi_{C M}\left(\vec{X}_{C M}\right)\right|^{2} \propto \delta^{(3)}\left(\vec{X}_{C M}\right)$. The hybrid potentials $\tilde{V}_{1,2}$ are related to the one- and two-body amplitudes (obtained by taking the non-relativistic limit of (12) and (18)) as follows:

$$
\begin{aligned}
\tilde{V}_{1}\left(\vec{q}_{W} ; \vec{x}_{i}\right) & =-e^{i \vec{q}_{W} \cdot \vec{x}_{i}} \bar{M}_{1, W}\left(\vec{q}_{W}\right) \\
\tilde{V}_{2}\left(\vec{q}_{W} ; \vec{x}_{i}, \vec{x}_{j}\right) & =-\int \frac{d \vec{q}_{i}}{(2 \pi)^{3}} \frac{d \vec{q}_{j}}{(2 \pi)^{3}} e^{-i \vec{q}_{i} \cdot \vec{x}_{i}} e^{-i \vec{q}_{j} \cdot \vec{x}_{j}}(2 \pi)^{3} \delta^{(3)}\left(\vec{q}_{i}+\vec{q}_{j}+\vec{q}_{W}\right) \bar{M}_{2, W}\left(\vec{q}_{i}, \vec{q}_{j}, \vec{q}_{W}\right)
\end{aligned}
$$

The explicit form of the two-body potentials at $\vec{q}_{W}=0$ is given by:

$$
\begin{aligned}
& \tilde{V}_{2}^{(\pi \pi)}\left(0 ; \vec{x}_{i}, \vec{x}_{j}\right)=-\frac{\lambda_{+}}{v \Lambda_{\mathrm{np}}^{2}} \frac{g_{A}^{2} m_{\pi}^{3}}{96 \pi F_{\pi}^{2}} O_{\pi \pi}(i, j) \\
& \tilde{V}_{2}^{(\eta \eta)}\left(0 ; \vec{x}_{i}, \vec{x}_{j}\right)=-\frac{1}{v \Lambda_{\mathrm{np}}^{2}} \frac{g_{A}^{2} m_{\eta}}{288 \pi F_{\pi}^{2}}\left(\frac{4 \alpha-1}{\sqrt{3}}\right)^{2}\left[m_{\pi}^{2} \lambda_{+}+4\left(M_{K}^{2}-\frac{1}{2} m_{\pi}^{2}\right) \lambda_{s}\right] O_{\eta \eta}(i, j)
\end{aligned}
$$

with

$$
\begin{aligned}
& O_{\pi \pi}(i, j)=-\frac{1}{x_{\pi}}\left(F_{1}\left(x_{\pi}\right) \delta_{a b}+F_{2}\left(x_{\pi}\right) T_{a b}\right)\left(\boldsymbol{\sigma}_{i}^{a} \boldsymbol{\sigma}_{j}^{b}\right) \otimes\left(\boldsymbol{\tau}_{i} \cdot \boldsymbol{\tau}_{j}\right), \\
& O_{\eta \eta}(i, j)=-\frac{1}{x_{\eta}}\left(F_{1}\left(x_{\eta}\right) \delta_{a b}+F_{2}\left(x_{\eta}\right) T_{a b}\right)\left(\boldsymbol{\sigma}_{i}^{a} \boldsymbol{\sigma}_{j}^{b}\right) \otimes(\mathbb{I}),
\end{aligned}
$$

and the definitions $\boldsymbol{\rho}=\boldsymbol{x}_{i}-\boldsymbol{x}_{j}, T_{i j}=3 \hat{\boldsymbol{\rho}}_{i} \hat{\boldsymbol{\rho}}_{j}-\delta_{i j}, x_{\pi}=m_{\pi}|\boldsymbol{\rho}|, x_{\eta}=m_{\eta}|\boldsymbol{\rho}|, F_{1}(x)=\mathrm{e}^{-x}(x-$ 2), $F_{2}(x)=\mathrm{e}^{-x}(x+1)$ [51, 26].

Evaluating the above matrix elements requires knowledge of the nuclear many body wave function $\left|\psi_{0}\right\rangle$. In particular, evaluating the one-body (23) and two-body contributions (24) requires knowledge of the one- and two-body nucleon densities in the ground state. For typical nuclei involved in DM direct detection experiments such as Ge or Xe the relevant wave function cannot be obtained from first principles and different models have to be used.

The one-body contributions to the WIMP-nucleus amplitude can be evaluated in a straightforward way noting that, given the one-body potential from (25), the matrix element (23) factorizes in the product of nucleon scalar form factors and nuclear form factor (the Fourier transform of the one-body nucleon densities). Denoting the latter by $F_{n, p}\left(\left|\vec{q}_{W}\right|^{2}\right)$ and assuming that neutron and proton densities are equal, $F_{n, p}\left(\left|\vec{q}_{W}\right|^{2}\right) \equiv F\left(\left|\vec{q}_{W}\right|^{2}\right)$, one finds:

$$
T_{1}=-F\left(\left|\vec{q}_{W}\right|^{2}\right)\left(Z f_{p}\left(\left|\vec{q}_{W}\right|^{2}\right)+(A-Z) f_{n}\left(\left|\vec{q}_{W}\right|^{2}\right)\right)
$$


with $f_{p, n}$ given in Eq. (13). The one-body density can be taken from phenomenology or from microscopic models, such as the nuclear shell model. We will use the exponential form [52] $F\left(E_{R}\right)=\operatorname{Exp}\left(-E_{R} /\left(2 E_{0}\right)\right)$ with $E_{R}=\left|\vec{q}_{W}\right|^{2} /\left(2 m_{A}\right), E_{0}=1.5 /\left(m_{A} R_{0}^{2}\right)$ and $R_{0}=[0.3+$ $\left.0.91\left(m_{A} / \mathrm{GeV}\right)^{1 / 3}\right] \times 10^{-13} \mathrm{~cm}$. We have checked that the results are stable if we use other parameterizations available in the literature [53, 52].

In order to calculate the two-body contribution $T_{2}$ (24) to the WIMP-nucleus amplitude, one needs the matrix elements

$$
\mathcal{N}_{M M}=\left\langle\psi_{0}\left|\sum_{i<j} O_{M M}(i, j)\right| \psi_{0}\right\rangle \quad M=\pi, \eta
$$

of the operators $O_{\pi \pi}$ and $O_{\eta \eta}$ defined in Eq. (29) and Eq. (30), in terms of which one has

$$
\begin{aligned}
& T_{2}^{(\pi \pi)}=-\frac{\lambda_{+}}{v \Lambda_{\mathrm{np}}^{2}} \frac{g_{A}^{2} m_{\pi}^{3}}{96 \pi F_{\pi}^{2}} \mathcal{N}_{\pi \pi} \\
& T_{2}^{(\eta \eta)}=-\frac{1}{v \Lambda_{\mathrm{np}}^{2}} \frac{g_{A}^{2} m_{\eta}}{288 \pi F_{\pi}^{2}}\left(\frac{4 \alpha-1}{\sqrt{3}}\right)^{2}\left[m_{\pi}^{2} \lambda_{+}+4\left(M_{K}^{2}-\frac{1}{2} m_{\pi}^{2}\right) \lambda_{s}\right] \mathcal{N}_{\eta \eta} .
\end{aligned}
$$

To evaluate the matrix elements $\mathcal{N}_{\pi \pi, \eta \eta}$ we use the NSM. In this framework, one assumes that the nucleons feel a mean external potential and occupy levels according to Pauli's exclusion principle. For the self-consistent potential, we use the harmonic oscillator with nucleusdependent frequency $\omega(A)$ empirically fit to data ${ }^{3}$. Given an arbitrary two-body potential $V_{i j}$ between nucleons $i$ and $j$, NSM allows to calculate the expectation value of the following Hamiltonian: $G=\sum_{i<j} V_{i j}$. For the simplest case of all closed shells (core-core matrix element), using the raising and lowering operator formalism the result for such expectation value in NSM equals:

$$
\langle c|G| c\rangle=\sum_{j 1 \leq j_{2}, J, T}(2 J+1)(2 T+1) V_{J T}\left(j_{1}, j_{2}, j_{1}, j_{2}\right)
$$

where $j_{i}$ represent the orbits of NSM, encoding quantum numbers $n, l, j$ of the orbit, $j_{1} \leq j_{2}$ is understood in the sense $E_{j_{1}} \leq E_{j_{2}}$, and finally $J$ runs $\left|j_{1}-j_{2}\right| \ldots j_{1}+j_{2}, T=0,1$. $V_{J T}$ is a two-body matrix element between the anti-symmetrized two-body wavefunction. Explicit expression for such two-body matrix element can be found in Ref. [55]. By computing the matrix elements for a number of closed-shell nuclei, we find the following scaling with $A$

$$
\mathcal{N}_{\pi \pi} \approx-0.91 A, \quad \mathcal{N}_{\eta \eta} \approx 0.0061 A
$$

For $\mathcal{N}_{\pi \pi}$ the scaling is consistent with $\mathcal{N}_{\pi \pi} \sim A$ found in [26]. The sign difference between $\mathcal{N}_{\pi \pi}$ and $\mathcal{N}_{\eta \eta}$ appears because for the pion exchange diagram the second term in the scalar form-factor $F_{1}(x)=(x-2) \exp (-x)$ dominates, while for $\eta \eta$ on the contrary, the first term dictates the sign. In order to understand the size difference one has to compare $m_{\pi} \mathcal{N}_{\pi \pi}$ to $m_{\eta} \mathcal{N}_{\eta \eta}$ since the meson mass $m_{M}$ has been factored out in the definitions of nuclear operators

\footnotetext{
${ }^{3}$ We use the following form for the harmonic oscillator frequency: $\omega(A)=\left(45 / A^{1 / 3}-25 / A^{2 / 3}\right) \mathrm{MeV}[54$. For the lower cutoff in the radial integrals we use $0.5 \mathrm{fm}$.
} 
$\mathcal{O}_{M M}$. The ratio of these numbers approximately equals 37 . This arises from a factor of 3 suppression for $\eta \eta$ operator due to different isospin structure and a factor of $\approx 12$ due to shorter distance potential for $\eta \eta$ compared to $\pi \pi$ exchange. Thus the expected value for the ratio $T_{2}^{(\eta \eta)} / T_{2}^{(\pi \pi)}$ is $1 / 37 \times 20 \times(4 \alpha-1)^{2} / 3 \approx 0.07$, where the factor of $\approx 20$ arises due to the strange Yukawa enhancement factor for the $\eta \eta$ operator.

\section{Phenomenology}

The differential WIMP-nucleus scattering rate per unit time and unit detector mass reads

$$
\frac{d R}{d E_{R}}=\frac{\kappa_{W} \rho_{W}}{\pi m_{W}}\left|\left[Z f_{p}\left(E_{R}\right)+(A-Z) f_{n}\left(E_{R}\right)\right] F\left(E_{R}\right)-T_{2}\left(E_{R}, A, Z\right)\right|^{2} \eta\left(E_{R}, m_{W}, m_{A}\right),
$$

where $E_{R}$ denotes the nuclear recoil energy, related to the momentum transfer squared via $-q_{W}^{2} \simeq\left|\vec{q}_{W}\right|^{2}=2 m_{A} E_{R}$ ( $m_{A}$ is the nucleus mass). The overall factor $\kappa_{W}$ in (37) depends on the nature of the DM particle. For example, for Dirac fermions $\kappa_{W}=1 / 2$, while for Majorana fermions $\kappa_{W}=2$. We denote by $m_{W}$ the WIMP mass and $\rho_{W}$ the local dark matter density (for which we use $\rho_{W}=0.3 \mathrm{GeV} / \mathrm{cm}^{3}$ ). Next, the WIMP-nucleus scattering amplitude is given by the sum of a one-body term involving the nucleon form factors $f_{p, n}\left(E_{R}\right)$ (see Eq. (13)) and nuclear form factor $F\left(E_{R}\right)$ (see discussion following (31)), and a two-body term $T_{2}\left(E_{R}, A, Z\right.$ ) (see Eq. (33) and Eq. (34)). Finally, the last factor in (37) involves an integral over the local DM velocity distribution $f(u)$ :

$$
\eta\left(E_{R}, m_{W}, m_{A}\right)=\int_{u_{\min }}^{u_{\mathrm{esc}}} \frac{f(u)}{u} \mathrm{~d}^{3} \boldsymbol{u}, \quad u_{\min }=\sqrt{\frac{m_{A} E_{R}}{2 \mu_{W A}^{2}}} \quad \mu_{W A}=\frac{m_{W} m_{A}}{m_{W}+m_{A}} .
$$

In our study we use for illustrative purposes a Maxwellian distribution with finite escape velocity (for which analytic expressions can be found in Ref. [19]), with input parameters $v_{0}=220 \mathrm{~km} / \mathrm{sec}, v_{\text {obs }}=233 \mathrm{~km} / \mathrm{sec}, v_{\text {esc }}=550 \mathrm{~km} / \mathrm{sec}[19]$.

Fixing the hadronic parameters to the central values of the ranges discussed in Section 4.1 , we can express the nucleon form factors and the two-body amplitude as linear combinations of the short-distance parameters $\lambda_{u . d . s, \Theta}$ defined above in Eq. (5). At zero momentum transfer the form factors read 4

$$
\begin{aligned}
& f_{p}(0) \equiv f_{p}=\frac{1}{v \Lambda_{\mathrm{np}}^{2}} \times\left(17.7 \lambda_{u}+24.5 \lambda_{d}+45.0 \lambda_{s}+938.3 \lambda_{\Theta}\right) \times \mathrm{MeV} \\
& f_{n}(0) \equiv f_{n}=\frac{1}{v \Lambda_{\mathrm{np}}^{2}} \times\left(12.2 \lambda_{u}+35.5 \lambda_{d}+45.0 \lambda_{s}+939.5 \lambda_{\Theta}\right) \times \mathrm{MeV}
\end{aligned}
$$

The energy-dependence of the form factors arising from loop corrections is very well approxi-

\footnotetext{
${ }^{4}$ The coefficients of $\lambda_{u, d}$ are proportional to $\sigma_{\pi N}$, while the coefficient of $\lambda_{s}$ is $\sigma_{s}$, so one can immediately assess the impact of hadronic uncertainties.
} 
mated by a linear form for $E_{R}<50 \mathrm{keV}::^{5}$

$$
\begin{aligned}
& f_{p}\left(E_{R}\right)-f_{p}(0) \simeq \frac{1}{v \Lambda_{\mathrm{np}}^{2}} \times\left[\left(-0.58 \lambda_{u}-0.96 \lambda_{d}-2.38 \lambda_{s}\right) \mathrm{MeV}\right] \times\left(\frac{A}{100}\right)\left(\frac{E_{R}}{50 \mathrm{KeV}}\right) \\
& f_{n}\left(E_{R}\right)-f_{n}(0) \simeq \frac{1}{v \Lambda_{\mathrm{np}}^{2}} \times\left[\left(-0.48 \lambda_{u}-1.16 \lambda_{d}-2.38 \lambda_{s}\right) \mathrm{MeV}\right] \times\left(\frac{A}{100}\right)\left(\frac{E_{R}}{50 \mathrm{KeV}}\right)
\end{aligned}
$$

Using the exact one-loop function or the above linear parameterization leads to differences in the rates at most of $0.1 \%$ (the largest deviations occurs for Xenon target and $m_{W}>100 \mathrm{GeV}$ ). Finally, the two-body amplitude is given by

$$
-T_{2}(0, A, A / 2)_{\text {closed shells }}^{\mathrm{NSM}}=\frac{1}{v \Lambda_{\mathrm{np}}^{2}} \times A \times\left(-0.48 \lambda_{u}-0.97 \lambda_{d}+0.089 \lambda_{s}\right) \times \mathrm{MeV},
$$

where we limited ourselves to closed shells, $Z=A-Z=A / 2$. While strictly speaking this formula is inapplicable for most target nuclei used in experiments, we will use it as a rough estimate of the two-body effect. An improved analysis should go beyond closed shells and include the dependence of the two-body amplitude on $E_{R}$. We leave this to future work.

The main novelty of our analysis stems from including in Eq. (37) the $E_{R}$-dependence of $f_{p, n}\left(E_{R}\right)$ and the two-body amplitude $T_{2}$, both of which arise to NLO in the chiral power counting. These long-distance QCD effects should be included in any consistent fit to direct DM detection searches because, as we show below, they can affect both the shape of the recoil spectrum and the total rates in a non-trivial way. We wish to emphasize here a few points:

- While the new effects are of the natural size expected by power counting (compare (39)(40) to (41), (42), and (43)), they become very important in those regions of parameter space where the leading order contribution to the WIMP-nucleus amplitude are suppressed (such as those realizing the so-called "isospin-violating dark matter" scenarios).

- Note that the NLO corrections depend on the recoil energy and have a different dependence on the short-distance parameters $\lambda_{i}$ than the LO contributions. As a result, the differential cross-section (37) does not factorize into a product of a cross-section $\sigma_{p} \times\left[Z+(A-Z) f_{n} / f_{p}\right]^{2}$, depending only on short-distance parameters $\lambda_{i}$, and a term depending only on long-distance QCD and nuclear effects. Still, for the differential cross-section the astrophysical dependence in $\eta$ factorizes. The scattering rate, however, obtained from integrating (37) over an energy window, no longer exhibits a factorization into a product of $\sigma_{p} \times\left[Z+(A-Z) f_{n} / f_{p}\right]^{2}$ with a term that is schematically astrophysical $\otimes$ nuclear and independent of the short-distance parameters. Using factorization to compare positive and null results of different direct-detection experiments, independent of assumptions about the DM velocity distribution, may have to be re-examined.

- Perhaps most importantly, our results show that the scalar-mediated WIMP-nucleus cross-section cannot be parameterized in terms of just two quantities, namely $f_{p}$ and

${ }^{5}$ The uncertainty in $\alpha=F /(F+D) \in[0.3,0.5]$ affects the coefficients of $\lambda_{u, d}$ at the $5 \%$ level, and the coefficient of $\lambda_{s}$ at the $20 \%$ level. 

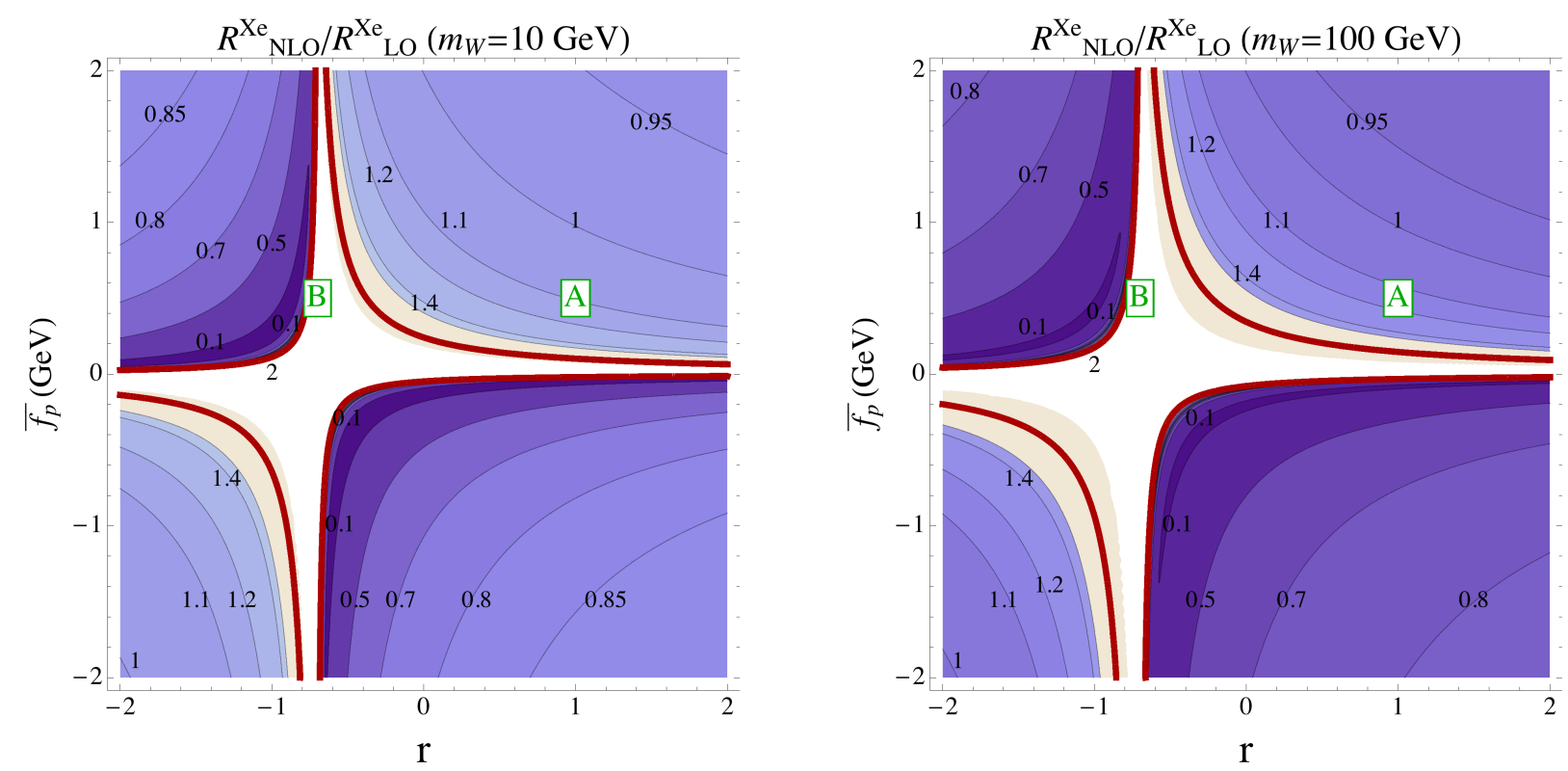

Figure 4: Contour plots of the NLO to LO integrated rates $R_{\mathrm{NLO}}^{\mathrm{Xe}} / R_{\mathrm{LO}}^{\mathrm{Xe}}$ on the $\left(r, \bar{f}_{p}\right)$ plane, at fixed $\lambda_{s} / \lambda_{\Theta}=1$, with $m_{W}=10 \mathrm{GeV}$ (left panel) and $m_{W}=100 \mathrm{GeV}$ (right panel). The solid red line corresponds to $R_{\mathrm{NLO}}^{\mathrm{Xe}} / R_{\mathrm{LO}}^{\mathrm{Xe}}=2$ and for all points inside the solid red line the NLO correction is more than $100 \%$.

$f_{n}$ or equivalently the WIMP-proton cross-section $\sigma_{p} \propto m_{p}^{2} f_{p}^{2}$ and the ratio $r=f_{n} / f_{p}$. Starting from the short-distance interaction of Eq. (11), the cross-section depends on four parameters (in one-to-one correspondence with $\lambda_{u, d, s, \Theta}$ ). This calls for a more general analysis of data, that takes into account these additional degrees of freedom.

A convenient choice of independent parameters, that matches onto the standard choice when neglecting NLO chiral corrections, is achieved as follows. First, we observe that $f_{n, p}\left(E_{R}\right)$ and $T_{2}\left(E_{R}, A, Z\right)$ are linear functions of $\lambda_{u, d, s, \Theta} / \Lambda_{\mathrm{np}}^{2}$ so that the rate is a homogeneous quadratic form in the $\lambda$ 's. Next, we can trade $\lambda_{u, d}$ for $f_{p}$ and $r=f_{n} / f_{p}$, and finally we can extract $\lambda_{\Theta}$ as an overall factor. In conclusion, the four parameters controlling the rate are: (1) $\lambda_{\Theta} /\left(v \Lambda_{\mathrm{np}}^{2}\right)$, which sets the overall normalization; (2) $f_{p}$, or equivalently ${ }^{6} \bar{f}_{p}=v \Lambda_{\mathrm{np}}^{2} f_{p} / \lambda_{\Theta}$; (3) $r=f_{n} / f_{p}$; and (4) $\lambda_{s} / \lambda_{\Theta}$. The rate has the form $R \sim\left(\lambda_{\Theta} /\left(v \Lambda_{\mathrm{np}}^{2}\right)\right)^{2} \times Q\left(f_{p}, r f_{p}, \lambda_{s} / \lambda_{\Theta}\right)$, where $Q(x, y, z)$ is a quadratic form in $x, y, z$. Neglecting NLO corrections, only two independent parameters survive, namely $f_{p}$ (or equivalently $\sigma_{p} \propto m_{p}^{2} f_{p}^{2}$ ) and $r=f_{n} / f_{p}$, and the rate takes the simplified form $R \sim f_{p}^{2}[Z+(A-Z) r]^{2}$. Note that any ratios of integrated rates only depend on three parameters: $\bar{f}_{p}, r$, and $\lambda_{s} / \lambda_{\theta}$, as the overall normalization cancels.

We illustrate the phenomenological implications of our new WIMP-nucleus amplitude parameterization in Figs. 4, 5, and 6.

- In Fig. 4 we present contour plots of the ratio of NLO to LO integrated rates $R_{\mathrm{NLO}} / R_{\mathrm{LO}}$ on the plane $\left(r, \bar{f}_{p}\right)$, fixing $\lambda_{s} / \lambda_{\Theta}=1$. We have chosen one representative target, Xenon,

\footnotetext{
${ }^{6}$ The convenience of this choice is apparent from equations Eq. 39 and Eq. (40). It is also clear that $\bar{f}_{p}$ has dimensions of energy.
} 

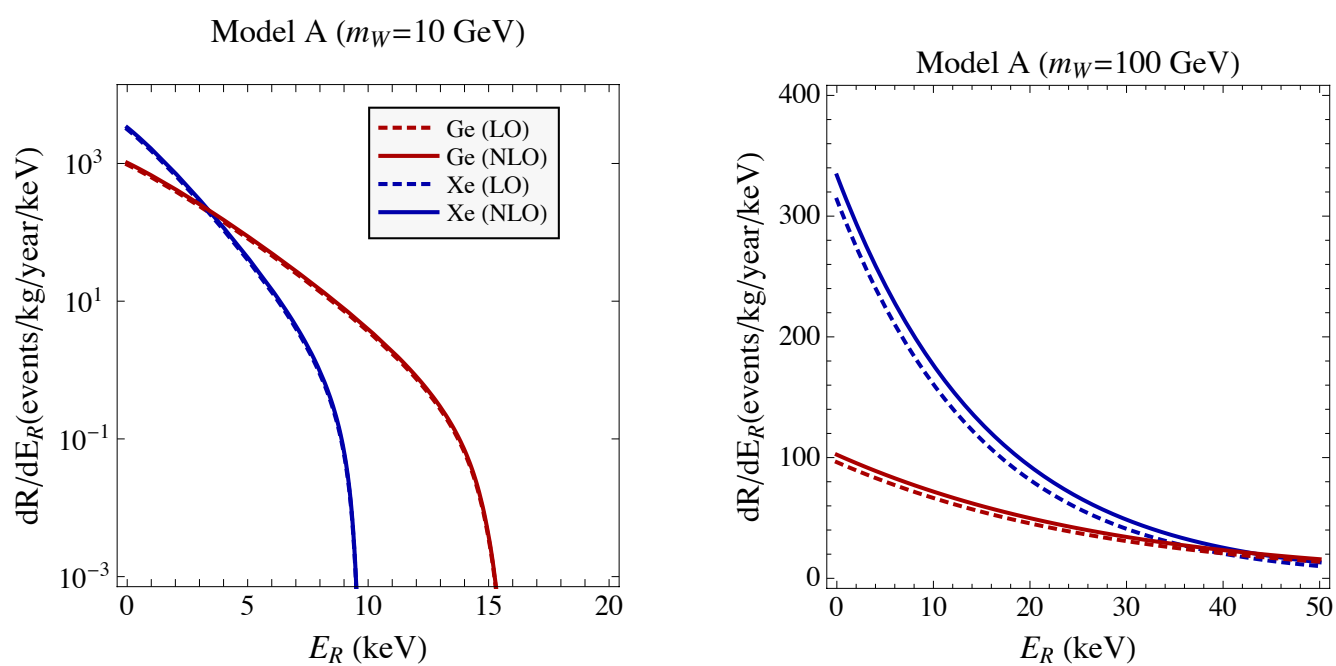

Model B $\left(m_{W}=10 \mathrm{GeV}\right)$
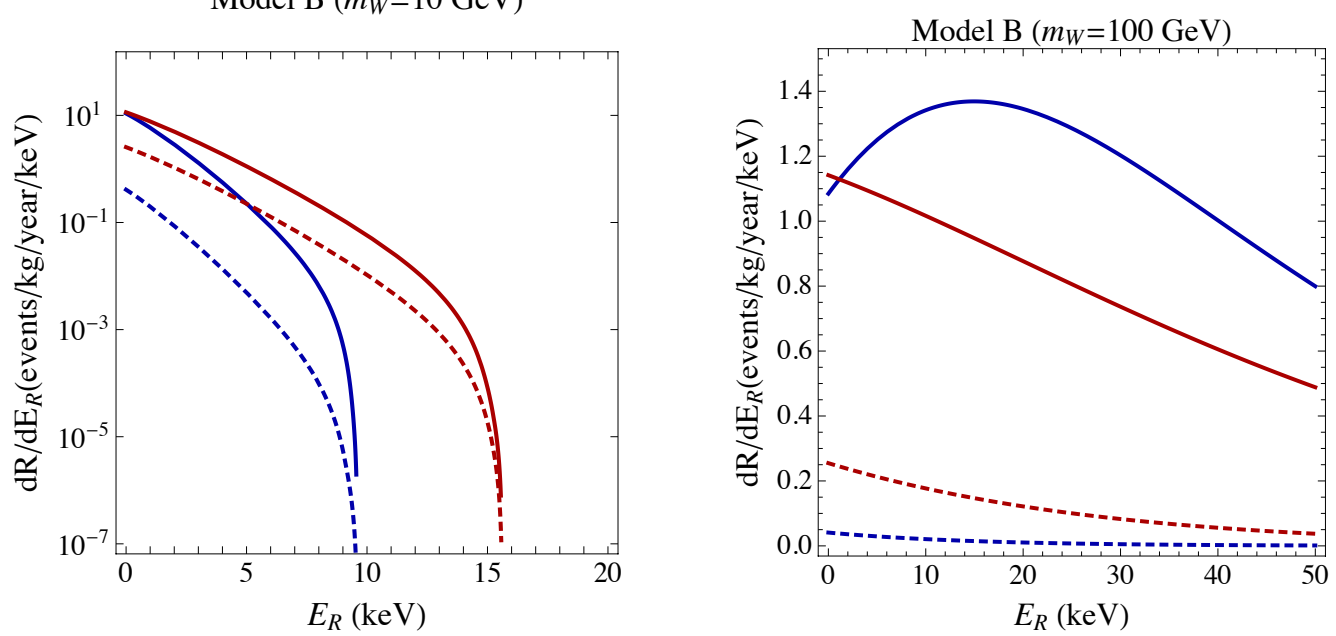

Figure 5: Recoil spectra for model A $\left(\bar{f}_{p}=0.5 \mathrm{GeV}, r=1, \lambda_{s, \Theta}=1\right.$, top panels $)$ and model $\mathrm{B}\left(\bar{f}_{p}=0.5 \mathrm{GeV}, r=-0.7, \lambda_{s, \Theta}=1\right.$ bottom panels), for both Xenon (blue lines) and Germanium (red lines) to LO (dashed lines) and NLO (solid lines).

for which we considered a weighted average of all naturally occurring isotopes and the integration region $E_{R} \in[8.4,44.6] \mathrm{keV}$ [3]. We plot results for two representative values of the WIMP mass $m_{W}=10 \mathrm{GeV}$ (left panel) and $m_{W}=100 \mathrm{GeV}$ (right panel). Qualitatively similar features arise for different choices of $\lambda_{s} / \lambda_{\Theta} \in[-50,50]$ and other target materials, such as Germanium. The plots clearly display the importance of NLO corrections whenever the LO rate vanishes or is highly suppressed, which happens for $f_{p}=0$ (for any finite $\left.r\right)$ and $Z+(A-Z) r=0(r \approx-0.7$ for Xenon isotopes). Along these singular directions the ratio $R_{\mathrm{NLO}} / R_{\mathrm{LO}}$ diverges or is highly enhanced. Moving away from these singular regions, the ratio $R_{\mathrm{NLO}} / R_{\mathrm{LO}}$ decreases, but corrections remain substantial over large regions of parameter space. We quantify this statement by highlighting in red the contours where $R_{\mathrm{NLO}} / R_{\mathrm{LO}}=2$ : within the region enclosed by these contours the fractional corrections to the rate exceed $100 \%$. 
- In Fig. 5 we illustrate the impact of chiral corrections on the recoil spectra, for two benchmark points in the $\left(r, \bar{f}_{p}\right)$ plane, model A where $R_{\mathrm{NLO}} / R_{\mathrm{LO}}-1 \sim \mathcal{O}(10 \%)$ (top panels), and model $\mathrm{B}$ where $R_{\mathrm{NLO}} / R_{\mathrm{LO}}$ is dramatically enhanced (bottom panels). In these plots we use $\Lambda_{\text {np }}=100 \mathrm{GeV}, v=246 \mathrm{GeV}$ and $\lambda_{\Theta}=1$ (the scaling of the rate with these parameters is trivial). The main message is that while for low-mass WIMP $\left(m_{W}=10 \mathrm{GeV}\right.$, left panels) the recoil spectrum gets mostly a normalization correction with no dramatic change in the shape regardless of the value of $r$, for larger WIMP masses $\left(m_{W}=100 \mathrm{GeV}\right.$, right panels) the recoil spectrum is considerably distorted when $Z+(A-Z) r \approx 0$. This result arises from the competition between the linearly rising $f_{n, p}\left(E_{R}\right)$ and the exponentially falling velocity integral $\eta\left(E_{R}, m_{W}, m_{A}\right)$, which cuts off at lower $E_{R}$ for lower $m_{W}$.

- Finally, in Fig. 6, we explore to what extent in our general framework for scalar-mediated WIMP-nucleus interactions we can reconcile the tension between CoGeNT [2, which favors a low-mass WIMP and XENON100 [3], which puts an upper limit on the rate in this mass region. The tension can be quantified as follows: for $m_{W}=10 \mathrm{GeV}$ the standard LO fit with $r=f_{n} / f_{p}=1$ implies [8] $\sigma_{p}^{\text {XENON100 }}<4 \times 10^{-43} \mathrm{~cm}^{2}$ and $\sigma_{p}^{\text {CoGeNT }}>4 \times 10^{-42} \mathrm{~cm}^{2}$ (assuming large contaminations in CoGeNT [8]), and hence $\sigma_{p}^{\mathrm{XENON} 100} / \sigma_{p}^{\mathrm{CoGeNT}}<0.1$. In turn, this can be converted into an upper bound on the ratio of integrated rates $R^{\mathrm{Xe}} / R^{\mathrm{Ge}}$ at $m_{W}=10 \mathrm{GeV}$, for any energy window. Using $E_{R} \in$ $[8.4,44.6] \mathrm{keV}$ [3] for Xe (XENON100) and $E_{R} \in[2.3,11.2] \mathrm{keV}$ [7] for Ge (CoGeNT) we find $R^{\mathrm{Xe}} / R^{\mathrm{Ge}}<2 \times 10^{-5}$.

In Fig. 6 we show contour plots of $R^{\mathrm{Xe}} / R^{\mathrm{Ge}}$ in the $\left(r, \bar{f}_{p}\right)$ plane to LO (top left panel) and $\mathrm{NLO}$ with $\lambda_{s} / \lambda_{\Theta}=1$ (top right panel). In these plots we also highlight in red the curves along which $R^{\mathrm{Xe}} / R^{\mathrm{Ge}}=2 \times 10^{-5}$. As seen from the top left panel, assuming LO cross-sections there is a narrow region around $r=-0.7$ consistent with experimental constraints. This is the well known regime of isospin violating dark matter (IVDM) [12, [13, 14, 15]. However, as expected from Fig. 4 and explicitly shown in the top right panel of Fig 6, along the $r=-0.7$ line the LO analysis cannot be trusted. Interestingly, our results show that to NLO there are still regions of parameter space consistent with $R^{\mathrm{Xe}} / R^{\mathrm{Ge}}<2 \times 10^{-5}$, which are non-trivial deformations of the narrow band around $r \approx-0.7$. In these regions, the NLO corrections provide a $90 \%$ suppression of the Xenon rates, i.e. $R_{\mathrm{NLO}}^{\mathrm{Xe}} / R_{\mathrm{LO}}^{\mathrm{Xe}}<0.1$, again pointing to the importance of the new effects. We have checked that even changing the energy-integration regions the same features emerge. For completeness, in the bottom panels of Fig. 6 we show the recoil spectra corresponding to two points in parameter space (marked as $\mathrm{C}$ and $\mathrm{D}$ in the top panels) consistent with $R^{\mathrm{Xe}} / R^{\mathrm{Ge}}<2 \times 10^{-5}$ to $\mathrm{NLO}$.

The main features of the results presented in Figs. 4, 5, and 6 are robust against changes in the hadronic matrix elements $\sigma_{q}^{(p, n)}$ and the low-energy constant $\alpha=F /(F+D)$. We have varied these inputs in the ranges presented in Section 4 and verified that the changes in Figs. 4, 5, and 6 are at the 5\% level at most. This uncertainty grows to about $20 \%$ level when $\lambda_{s} / \lambda_{\Theta} \gg 1$. A related important question for the phenomenology is: how robust is the one-loop ChPT calculation of the slope of the scalar form factors? The dispersive analysis of 

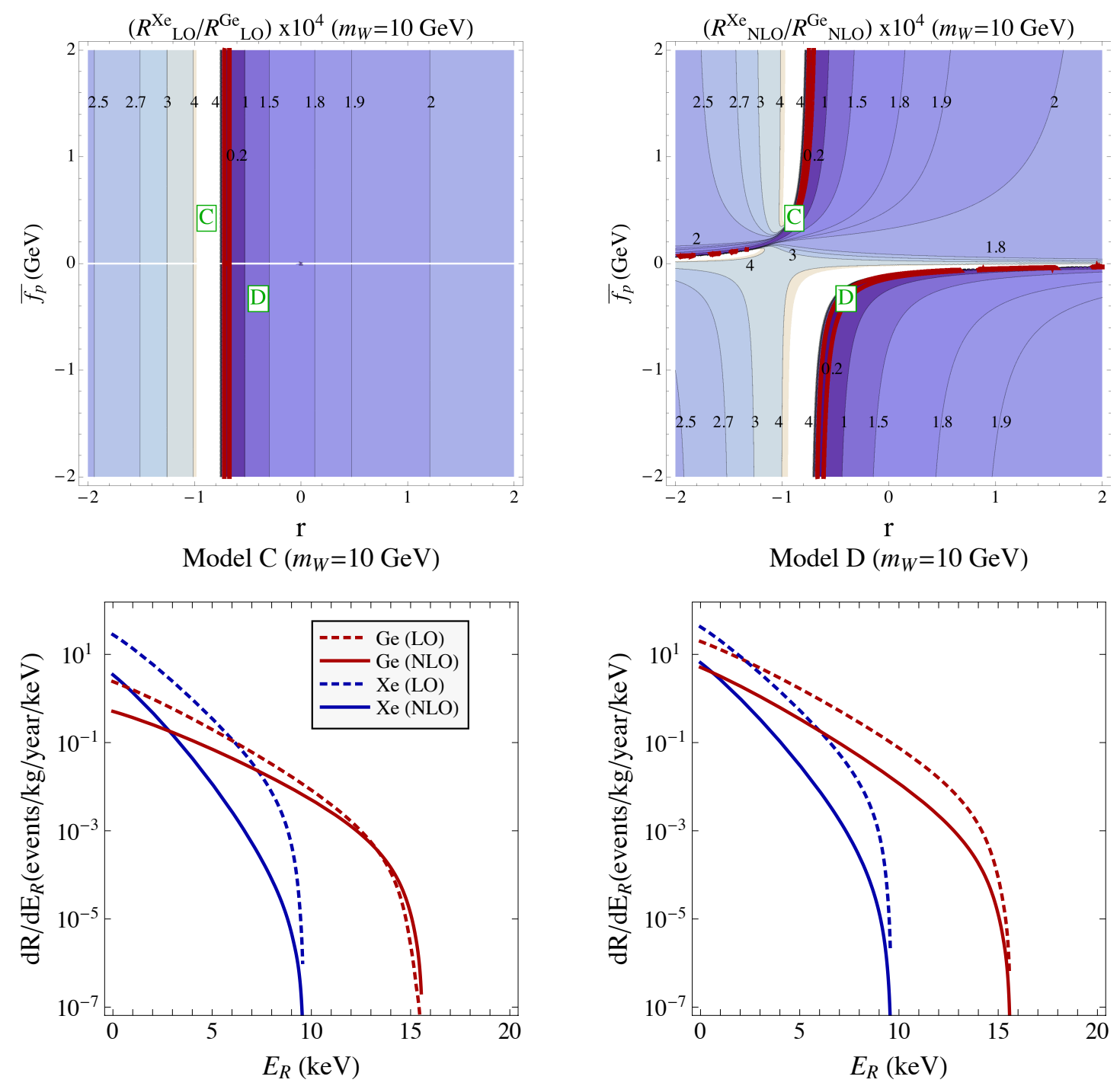

Figure 6: Top panels: contour plots of the ratio of Xenon and Germanium integrated rates $R^{\mathrm{Xe}} / R^{\mathrm{Ge}}$ on the $\left(r, \bar{f}_{p}\right)$ plane, at fixed $\lambda_{s} / \lambda_{\Theta}=1$ and $m_{W}=10 \mathrm{GeV}$ to LO (left panel) and NLO (right panel). Solid red lines on top panels represent the contour lines, where $R_{\mathrm{NLO}}^{\mathrm{Xe}} / R_{\mathrm{NLO}}^{\mathrm{Ge}}=2 \times 10^{-5}$. Everywhere inside the solid red lines the signal in CoGent is consistent with null signal in Xenon100. Bottom panels: recoil spectra for model $\mathrm{C}\left(\bar{f}_{p}=0.425 \mathrm{GeV}\right.$, $\left.r=-0.885, \lambda_{s, \Theta}=1\right)$ and model $\mathrm{D}\left(\bar{f}_{p}=-0.33 \mathrm{GeV}, r=-0.4, \lambda_{s, \Theta}=1\right)$, for both Xenon (blue lines) and Germanium (red lines) to LO (dashed lines) and NLO (solid lines).

Ref. [56] reveals that one-loop results severely under-estimate (by more than a factor of 2) the slope of the iso-scalar $(\bar{u} u+\bar{d} d)$ form factor. We expect that larger slopes will increase the impact of recoil-energy dependent form factors $\left(f_{n, p}\left(E_{R}\right)\right)$, reinforcing the conclusions of our work. Therefore, this issues deserves to be revisited in the future. 


\section{Conclusions}

We have applied systematic chiral effective theory methods to WIMP-nucleus interactions. Focusing on the case of scalar-mediated WIMP-quark interactions, but otherwise in a completely model-independent framework, we have worked out the NLO corrections to WIMP-nucleon interactions. A similar analysis can be done for pseudo-scalar, vector, pseudo-vector and tensor WIMP-quark interactions. We find that at NLO two types of effects enter. First, one loop diagrams generate recoil-energy dependent corrections to the single-nucleon scalar form factors. The second effect involves two-nucleon interactions with the WIMP at tree level. This generates a new two-body term in the WIMP-nucleus scattering amplitude. Our results for the modified rate formula (37) show that the scalar-mediated WIMP-nucleus cross-section cannot be parameterized in terms of just two quantities, namely $f_{p}$ and $f_{n}$ or equivalently the WIMP-proton cross-section $\sigma_{p} \propto m_{p}^{2} f_{p}^{2}$ and $r=f_{n} / f_{p}$. Two more parameters are needed for a complete description consistent with long-distance QCD effects.

In our model-independent scan of parameter space, we have found that the new effects become extremely important when the leading order contribution to the WIMP-nucleus amplitude is moderately to highly suppressed. We have identified the region of parameter space in which the fractional corrections are greater than $100 \%$, showing that it includes the socalled "isospin-violating dark matter" regime. We have also explored to what extent the tension between CoGeNT [2] and XENON100 [3], quantified by the ratio of integrated rates $R^{\mathrm{Xe}} / R^{\mathrm{Ge}}<2 \times 10^{-5}$, can be reconciled in our framework. Intriguingly, we find that there are regions of parameter space consistent with this constraint and we show that in these regions, the NLO corrections provide a $90 \%$ suppression of the Xenon rates, i.e. $R_{\mathrm{NLO}}^{\mathrm{Xe}} / R_{\mathrm{LO}}^{\mathrm{Xe}}<0.1$. Finally, we have also explored how the new corrections affect the recoil spectra, finding large distortions for regions in which the LO contribution is suppressed and for WIMP masses $m_{W} \geq 30 \mathrm{GeV}$.

Both the theoretical and phenomenological analysis presented here should be regarded as only the first step of a broader program. On the theory side, we can identify several areas where future work is highly desirable: (i) the extension of the ChPT analysis beyond scalar-mediated WIMP-quark interactions; (ii) an improved treatment of the slope of the single-nucleon scalar form factors; (iii) an improved analysis of the two-nucleon matrix element, that goes beyond closed shells and includes the dependence on the recoil energy $E_{R}$. On the phenomenology side, we have presented a few illustrations of how our new results affect direct DM detection, without any attempt to a complete analysis. We argue, however, that our results call for a new model-independent analysis of direct DM search data that properly includes long-distance QCD effects, because they can affect both the shape and normalization of the recoil spectrum.

\section{Acknowledgements}

We have benefited from many helpful discussions with Joe Carlson, Joe Ginocchio, and Anna Hayes. We also thank John Donoghue and Rocco Schiavilla for discussions and Alberto Aparici for collaboration at an early stage of this work. This work is supported by the DOE Office of Science and the LDRD program at Los Alamos. 


\section{References}

[1] R. Bernabei et al., DAMA Collaboration. First results from DAMA/LIBRA and the combined results with DAMA/NaI. Eur. Phys. J., C56:333-355, 2008, doi:10.1140/epjc/ s10052-008-0662-y, arXiv:0804.2741.

[2] C.E. Aalseth, P.S. Barbeau, J. Colaresi, J.I. Collar, J. Diaz Leon, et al. Search for an Annual Modulation in a P-type Point Contact Germanium Dark Matter Detector. Phys. Rev. Lett., 107:141301, 2011, doi:10.1103/PhysRevLett.107.141301, arXiv:1106.0650.

[3] E. Aprile et al., XENON100 Collaboration. Dark Matter Results from 100 Live Days of XENON100Data.Phys. Rev. Lett., 107:131302, 2011, doi:10.1103/PhysRevLett.107.131302, arXiv:1104.2549.

[4] J. Angle et al., XENON10 Collaboration. A search for light dark matter in XENON10 data. Phys. Rev. Lett., 107:051301, 2011, doi:10.1103/PhysRevLett.107.051301, arXiv:1104.3088.

[5] Z. Ahmed et al., CDMS Collaboration. Search for annual modulation in low-energy CDMS-II data. 2012, arXiv:1203.1309.

[6] Z. Ahmed et al., CDMS-II Collaboration.Results from a Low-Energy Analysis of the CDMS II Germanium Data.Phys. Rev. Lett., 106:131302, 2011, doi:10.1103/PhysRevLett.106.131302, arXiv:1011.2482.

[7] Marco Farina, Duccio Pappadopulo, Alessandro Strumia, and Tomer Volansky. Can CoGeNT and DAMA Modulations Be Due to Dark Matter? JCAP, 1111:010, 2011, doi:10.1088/1475-7516/2011/11/010, arXiv:1107.0715.

[8] Joachim Kopp, Thomas Schwetz, and Jure Zupan.Light Dark Matter in the light of CRESSTII. JCAP, 1203:001, 2012, doi:10.1088/1475-7516/2012/03/001, arXiv:1110.2721.

[9] David Tucker-Smith and Neal Weiner. Inelastic dark matter. Phys. Rev., D64:043502, 2001, doi:10.1103/PhysRevD.64.043502, arXiv:hep-ph/0101138.

[10] Peter W. Graham, Roni Harnik, Surjeet Rajendran, and Prashant Saraswat. Exothermic Dark Matter. Phys. Rev., D82:063512, 2010, doi:10.1103/PhysRevD.82.063512, arXiv:1004.0937.

[11] James M. Cline, Andrew R. Frey, and Fang Chen. Metastable dark matter mechanisms for INTEGRAL $511 \mathrm{keV} \gamma$ rays and DAMA/CoGeNT events. Phys. Rev., D83:083511, 2011, doi:10.1103/PhysRevD.83.083511, arXiv:1008.1784.

[12] Andriy Kurylov and Marc Kamionkowski. Generalized analysis of weakly interacting massive particle searches. Phys. Rev., D69:063503, 2004, doi:10.1103/PhysRevD.69.063503, arXiv:hep-ph/0307185. 
[13] F. Giuliani. Are direct search experiments sensitive to all spin-independent WIMP candidates? Phys. Rev. Lett., 95:101301, 2005, doi:10.1103/PhysRevLett.95.101301, arXiv:hep-ph/0504157.

[14] Spencer Chang, Jia Liu, Aaron Pierce, Neal Weiner, and Itay Yavin.CoGeNT Interpretations. JCAP, 1008:018, 2010, doi:10.1088/1475-7516/2010/08/018, arXiv:1004.0697.

[15] Jonathan L. Feng, Jason Kumar, Danny Marfatia, and David Sanford. Isospin-Violating Dark Matter. Phys. Lett., B703:124-127, 2011, doi:10.1016/j.physletb.2011.07.083, arXiv:1102.4331.

[16] Brian Feldstein, A. Liam Fitzpatrick, and Emanuel Katz. Form Factor Dark Matter. JCAP, 1001:020, 2010, doi:10.1088/1475-7516/2010/01/020, arXiv:0908.2991.

[17] Spencer Chang, Aaron Pierce, and Neal Weiner. Momentum Dependent Dark Matter Scattering.JCAP, 1001:006, 2010, doi:10.1088/1475-7516/2010/01/006, arXiv:0908.3192.

[18] Yang Bai and Patrick J. Fox.Resonant Dark Matter.JHEP, 0911:052, 2009, doi:10.1088/11266708/2009/11/052, arXiv:0909.2900.

[19] Christopher Savage, Katherine Freese, and Paolo Gondolo. Annual Modulation of Dark Matter in the Presence of Streams. Phys. Rev., D74:043531, 2006, doi:10.1103/ PhysRevD.74.043531, arXiv:astro-ph/0607121.

[20] Mariangela Lisanti, Louis E. Strigari, Jay G. Wacker, and Risa H. Wechsler. The Dark Matter at the End of the Galaxy. Phys. Rev., D83:023519, 2011, doi:10.1103/PhysRevD.83.023519, arXiv:1010.4300.

[21] Patrick J. Fox, Jia Liu, and Neal Weiner. Integrating Out Astrophysical Uncertainties. Phys. Rev., D83:103514, 2011, doi:10.1103/PhysRevD.83.103514, arXiv:1011.1915.

[22] Mads T. Frandsen, Felix Kahlhoefer, Christopher McCabe, Subir Sarkar, and Kai SchmidtHoberg. Resolving astrophysical uncertainties in dark matter direct detection. JCAP, 1201:024, 2012, doi:10.1088/1475-7516/2012/01/024, arXiv:1111.0292. 31 pages, 15 figures. v2: matches published version.

[23] Juan Herrero-Garcia, Thomas Schwetz, and Jure Zupan. Astrophysics independent bounds on the annual modulation of dark matter signals. 2012, arXiv:1205.0134.

[24] JiJi Fan, Matthew Reece, and Lian-Tao Wang. Non-relativistic effective theory of dark matter direct detection. JCAP, 1011:042, 2010, doi:10.1088/1475-7516/2010/11/042, arXiv:1008.1591.

[25] A. Liam Fitzpatrick, Wick Haxton, Emanuel Katz, Nicholas Lubbers, and Yiming Xu. The Effective Field Theory of Dark Matter Direct Detection. 2012, arXiv:1203.3542.

[26] Gary Prezeau, Andriy Kurylov, Marc Kamionkowski, and Petr Vogel. New contribution to wimp-nucleus scattering. Phys. Rev. Lett., 91:231301, 2003, doi:10.1103/PhysRevLett.91.231301, arXiv:astro-ph/0309115. 
[27] Mikhail A. Shifman, A.I. Vainshtein, and Valentin I. Zakharov. Remarks on Higgs Boson Interactions with Nucleons. Phys. Lett., B78:443, 1978, doi:10.1016/0370-2693(78)90481-1.

[28] Steven Weinberg. Phenomenological Lagrangians. Physica, A96:327, 1979.

[29] J. Gasser and H. Leutwyler. Chiral Perturbation Theory to One Loop. Annals Phys., 158:142, 1984, doi:10.1016/0003-4916(84)90242-2.

[30] J. Gasser and H. Leutwyler. Chiral Perturbation Theory: Expansions in the Mass of the Strange Quark. Nucl. Phys., B250:465, 1985, doi:10.1016/0550-3213(85)90492-4.

[31] V. Bernard, Norbert Kaiser, and Ulf-G. Meissner. Chiral dynamics in nucleons and nuclei. Int. J. Mod. Phys., E4:193-346, 1995, doi:10.1142/S0218301395000092, arXiv:hep-ph/9501384.

[32] Paulo F. Bedaque and Ubirajara van Kolck. Effective field theory for few nucleon systems. Ann. Rev. Nucl. Part. Sci., 52:339-396, 2002, doi:10.1146/annurev.nucl.52.050102.090637, arXiv:nucl-th/0203055.

[33] Andreas S. Kronfeld. Twenty-first Century Lattice Gauge Theory: Results from the QCD Lagrangian. 2012, arXiv:1203.1204.

[34] V. Cirigliano, M Graesser, and G. Ovanesyan. In preparation. 2012.

[35] Elizabeth Ellen Jenkins and Aneesh V. Manohar. Baryon chiral perturbation theory using a heavy fermion Lagrangian. Phys. Lett., B255:558-562, 1991, doi:10.1016/03702693(91)90266-S.

[36] Steven Weinberg. Nuclear forces from chiral Lagrangians. Phys. Lett., B251:288-292, 1990, doi:10.1016/0370-2693(90)90938-3.

[37] Steven Weinberg. Effective chiral Lagrangians for nucleon - pion interactions and nuclear forces. Nucl. Phys., B363:3-18, 1991, doi:10.1016/0550-3213(91)90231-L.

[38] David B. Kaplan, Martin J. Savage, and Mark B. Wise. Nucleon - nucleon scattering from effective field theory. Nucl. Phys., B478:629-659, 1996, doi:10.1016/0550-3213(96)00357-4, arXiv:nucl-th/9605002.

[39] John F. Donoghue and H. Leutwyler. Energy and momentum in chiral theories. Z. Phys., C52:343-351, 1991, doi:10.1007/BF01560453.

[40] Achille Corsetti and Pran Nath. Gaugino mass nonuniversality and dark matter in SUGRA, strings and D-brane models. Phys. Rev., D64:125010, 2001, doi:10.1103/ PhysRevD.64.125010, arXiv:hep-ph/0003186.

[41] B. Borasoy and Ulf-G. Meissner. Chiral expansion of baryon masses and sigma terms. Annals Phys., 254:192-232, 1997, doi:10.1006/aphy.1996.5630, arXiv: hep-ph/9607432. 
[42] M.M. Pavan, I.I. Strakovsky, R.L. Workman, and R.A. Arndt. The Pion nucleon Sigma term is definitely large: Results from a G. W. U. analysis of pi nucleon scattering data. PiN Newslett., 16:110-115, 2002, arXiv:hep-ph/0111066.

[43] H. Ohki, H. Fukaya, S. Hashimoto, T. Kaneko, H. Matsufuru, et al. Nucleon sigma term and strange quark content from lattice QCD with exact chiral symmetry. Phys. Rev., D78:054502, 2008, doi:10.1103/PhysRevD.78.054502, arXiv:0806.4744.

[44] R.D. Young and A.W. Thomas. Octet baryon masses and sigma terms from an SU(3) chiral extrapolation. Phys. Rev., D81:014503, 2010, doi:10.1103/PhysRevD.81.014503, arXiv:0901.3310.

[45] D. Toussaint and W. Freeman, MILC Collaboration. The Strange quark condensate in the nucleon in 2+1 flavor QCD. Phys. Rev. Lett., 103:122002, 2009, doi:10.1103/ PhysRevLett.103.122002, arXiv:0905.2432.

[46] K. Takeda et al., JLQCD collaboration. Nucleon strange quark content from two-flavor lattice QCD with exact chiral symmetry. Phys. Rev., D83:114506, 2011, doi:10.1103/ PhysRevD.83.114506, arXiv:1011.1964.

[47] S. Durr, Z. Fodor, T. Hemmert, C. Hoelbling, J. Frison, et al. Sigma term and strangeness content of octet baryons. Phys. Rev., D85:014509, 2012, doi:10.1103/PhysRevD.85.014509, arXiv:1109.4265.

[48] R. Horsley, Y. Nakamura, H. Perlt, D. Pleiter, P.E.L. Rakow, et al. Hyperon sigma terms for 2+1 quark flavours. Phys. Rev., D85:034506, 2012, doi:10.1103/PhysRevD.85.034506, arXiv:1110.4971.

[49] Hai-Yang Cheng. Low-energy interactions of scalar and pseudoscalar Higgs bosons with baryons. Phys. Lett., B219:347, 1989, doi:10.1016/0370-2693(89)90402-4.

[50] K. Nakamura et al., Particle Data Group. Review of particle physics. J. Phys. G, G37:075021, 2010, doi:10.1088/0954-3899/37/7A/075021.

[51] J.D. Vergados. Pion double charge exchange contribution to neutrinoless double beta decay. Phys. Rev., D25:914, 1982, doi:10.1103/PhysRevD.25.914.

[52] Gerard Jungman, Marc Kamionkowski, and Kim Griest.Supersymmetric dark matter.Phys. Rept., 267:195-373, 1996, doi:10.1016/0370-1573(95)00058-5, arXiv:hep-ph/9506380.

[53] J. Engel, S. Pittel, and P. Vogel. Nuclear physics of dark matter detection. Int. J. Mod. Phys., E1:1-37, 1992, doi:10.1142/S0218301392000023.

[54] Anna Hayes. Private communications.

[55] B. Castel and I.S. Towner. Modern Theories of Nuclear Moments. Oxford Studies in Nuclear Physics. 
[56] J. Gasser, H. Leutwyler, and M.E. Sainio. Form-factor of the sigma term. Phys. Lett., B253:260-264, 1991, doi:10.1016/0370-2693(91)91394-B. 